\title{
Tracking Moving Objects based on Background Subtraction using Kalman Filter
}

\author{
M.Arief Soeleman ${ }^{1}$, D. Raniasti ${ }^{2}$, Karis $^{3}$,Muljono ${ }^{4}$, Muslih $^{5}$, RA. Pramunendar ${ }^{6}$ \\ \{arief22208@gmail.com ${ }^{1}$,raniasti@gmail.com ${ }^{2}$,kariswidyatmoko@gmail.com ${ }^{3}$ \} \\ Faculty of Computer Science, Dian Nuswantoro University ${ }^{123456}$
}

\begin{abstract}
Detection and tracking of human objects is one of the important studies in improving the ability of the surveillance system. The aim of this study is to measure and analyze the application of background subtraction method and the filter capability of detecting human objects to trace human movements through CCTV video-based. This research applies background subtraction method to detect moving object, assisted with median filter and morphology operation which aims to get good results on objects that have been detected. Kalman filter is applied to the process of tracking object movementt. Performance evaluation is performed by using means square error to determine the performance of Kalman filter method.
\end{abstract}

Keywords: detection; tracking, background subtraction, kalman filter.

\section{Introduction}

Monitoring system [1] is a system which is extensively applied to perform monitoring functions periodically to obtain the desired information, particularly for moving objects. Results of the system have significant role in improving various aspects, that is identification [2], information, security, level of productivity, and performance. The example of monitoring system is the motion object detection application. Detection of moving objects is an object detector which define or extract information from objects in a frame sequence. Detection of human objects both indoors and outdoors is the sample of object detection applications

Detection of human objects is a significant aim of the research in order to improve the ability of the surveillance system in public spaces. In general, the mechanism of detecting human objects adapts the observing method in the real world through the human sight, starting from learning framesequences and videos to obtain results that humans can do.

Besides, detection of human objects attemps to imitate the workings of human visual system which is currently known as computer vision technology. Several studies have been performed in order to apply techniques or methods to detect human objects. One of method is background subtraction [3], [4], [5], [6] for moving detection in a static environment. In this study, researchers perform the application of background subtraction methods to detect human objects in video files.

Object detection has some basic formation, object detection is made from modeling the background and foreground. This modeling aims to distinguish pixels which will be the foreground-background. In the foreground-background modeling algorithm, the subtraction method or the difference of input frame is perfromed through background to obtain the foreground or moving objects. In prevoius studies, the mathematical modeling of object ICCSET 2018, October 25-26, Kudus, Indonesia

Copyright (C) 2018 EAI

DOI 10.4108/eai.24-10-2018.2280505 
detection has been divided into four principal quantities, using statistical methods to separate the background and foreground, examining for vectors representing the background and foreground, introducing the foreground (objects) with template matching, and applying the initiation of foreground features which based on uniqueness resulting from the transformation of frequency domain. In this paper the kalman filter will be applied to carry the performance improvements out in object tracking.

\section{Related Research}

Irene's research in [7] performed a combination of Mean-shift and Kalman filters. This combination is assembled to obtain the power of the two algorithms aiming the mean-shift to perform tracking when there are no objects blocking the movement. When oclusion occurs, kalman filter is applied as a combination tracking.

In another study, Pouya in [8] proposed a Kalman filter method for tracking parts of the human body, by predicting the position and limbs. This method successfully identifies by various poses and conditions obstructed by objects.

In his paper, Dali Shu [9] conducted a finger print data collection process with Kalman filter method to reduce noise, up and down signal and 2D signal mapping for fingerprints. The application of Kalman filter method, it results in the form of speed in the gaussian weight, speed and track the target more accurately

\section{Methodology}

This resesarch will have few steps in the processing, which are reading the frame video, moving object detection process by background, detected object filtration process by median filter, morphology operation, tracking by kalman filter. The initial process is reading the video frame in order to process the entire image on the video, then the background subtraction method is applied to detect the moving object, followed by the median filter operation, and the morphological operation. At each stage and operations performed, it will determine certain values which eventually detection tracking of human objects will be accomplished by the Kalman filter method. Details of the process stages accomplished are as follows:

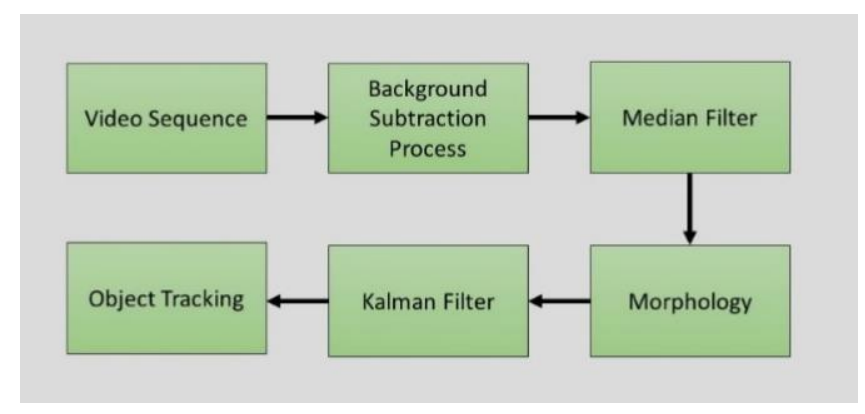

Fig. 1. Proposed method. 


\subsection{Background subtraction}

Background subtraction in [10] is a process to detect movement or significant differences which occur within the video frame compared to reference images. Background subtraction aims to separate objects and background so that the motion of an object is detected. Its application aims to produce a sequence of frames from a video and detect all foreground objects.. Description of the approach existed of background subtraction is to detect foreground objects as differences between the current frame and background image from a static screen. In the background subtraction, the moving color image is first converted into a gray or grayscale image so that it can be converted into binary imagery. The initial frame which have no moving object from a moving image that has been converted into binary image will be cleared from noise to produce a stable and sensitive background to changes in the foreground.

$$
F B(x, y, n)=\left\{\begin{array}{c}
1, \text { if }|I(x, y, n)-I(x, y, n-1)| \geq \alpha \\
0, \text { otherwise }
\end{array}\right.
$$

This approach is very sensitive to threshold $(\alpha)$, so that the threshold can be applied to adjust the sensitivity of a camera in capturing video frames.

\subsection{Median Filter}

Median filter [14] is non-linear filters applied to reduce noise in the image. It is included in the order-statistical filter, which is a spatial filter that works based on the order of pixels in the area covered by the filter. In the median filter, a mask containing an odd number of pixels is shifted dots per point in the entire image area. The values are sorted and then the median value is calculated. This value will replace the pixel value in the center of the mask with the size of $3 \times 3$.

\subsection{Morphology}

Morphological operation [11] is applied using the object shape as a guide in processing which aims to obtain better object segmentation results. The value of each pixel in the digital image results is obtained through a comparison process between corresponding pixels in the digital image input with neighboring pixels. The morphological operation performed is the process of erosion and dilation. Dilation is the process of adding pixels to the object edge in the digital image input, while erosion is the process of reducing pixels at the object edge. The number of pixels added or subtracted from the object edge in the digital image input depends on the size and shape of the structural elements used.

\subsection{Kalman Filter}

The Kalman filter [12] is a recursive estimator. It means that only the state estimation of the previous time step and current measurement is needed to calculate the estimation of current condition. Thus, no observation history or estimation needed. Kalman filters have two distinctive features. Mathematical model explained in terms of the concept of state-space is one of the features. The equation is explained below: 
And

$$
z_{k}=G_{k} x_{k}+n_{k}
$$

$$
x_{k}=F_{k} x_{k-1}+m_{k-1}
$$

Where $G_{k}$ and $F_{k}$ are user matrices defining the linear relationship

$$
x_{k \mid k-1}=F_{k} \hat{x}_{k-1}
$$

$$
\mathrm{P}_{\mathrm{k} \mid \mathrm{k}-1}=\mathrm{F}_{\mathrm{k}} \mathrm{P}_{\mathrm{k}-1}-\mathrm{F}_{\mathrm{k}}^{\prime}+\mathrm{Q}_{\mathrm{k}}
$$

We can compute the predicted measurement as

$$
\hat{z}_{k}=G_{k} \hat{x}_{k \mid k-1}
$$

The new measurement $\mathrm{z}_{\mathrm{k}}$ can derive the mean residual

The residual covariance

$$
\hat{\mathrm{r}}_{\mathrm{k}}=\mathrm{z}_{\mathrm{k}}-\hat{\mathrm{z}}_{\mathrm{k}}
$$

$$
\mathrm{S}_{\mathrm{k}}=\mathrm{G}_{\mathrm{k}} \mathrm{P}_{\mathrm{k} \mid \mathrm{k}-1} \mathrm{G}_{\mathrm{k}}^{\prime}+\mathrm{R}_{\mathrm{k}}
$$

And $\mathrm{K}_{\mathrm{k}}$ the Kalman gain as,

$$
\mathrm{K}_{\mathrm{k}}=\mathrm{P}_{\mathrm{k} \mid \mathrm{k}-1} \mathrm{G}_{\mathrm{k}}^{\prime} \mathrm{S}_{\mathrm{k}}^{-1}
$$

Finally, to complete recursion as the mean estimate

$$
\hat{\mathrm{x}}_{\mathrm{k}}=\hat{\mathrm{x}}_{\mathrm{k} \mid \mathrm{k}-1} \mathrm{~K}_{\mathrm{k}} \hat{\mathrm{r}}_{\mathrm{k}}
$$

And the posterior covariance

$$
P_{k}=\left(I-K_{k}-G_{k}\right) P_{k \mid k-1}
$$

\section{Experimental results}

In this experiment, we used MATLAB 2017 PC with Intel i5 M 200 processor with 4GB of memory. The video data is used by two datasets to test the reliability of the background subtraction method and kalman filter in detecting and tracking the moving object. 


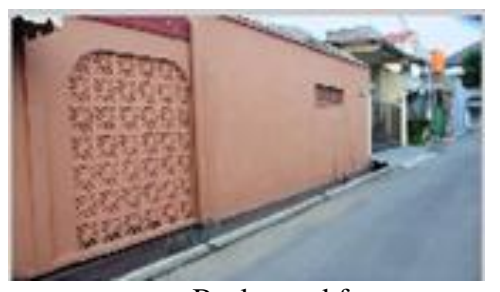

a. Backgroud frame



c. Result Subtraction

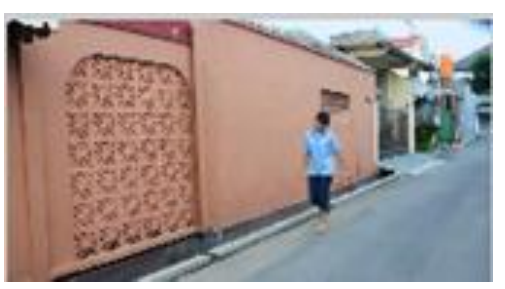

b. Frame 56 with object

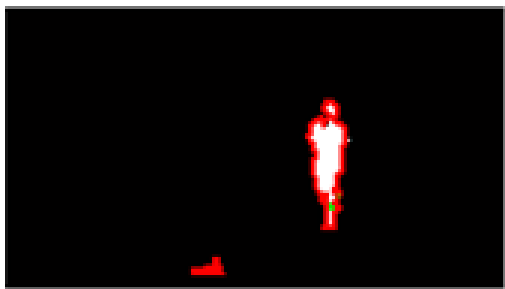

d. Blob detection

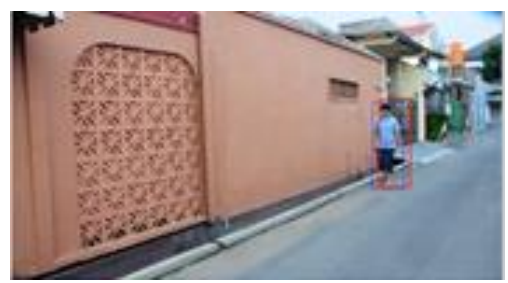

e. Object Tracking with kalman Filter

Fig. 2. Result detection object with background subtraction. 


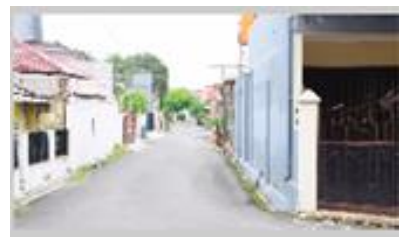

a. Background frame

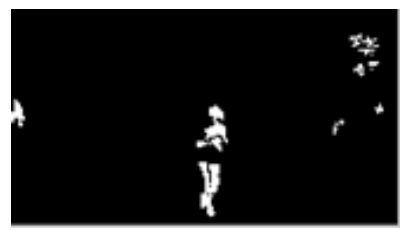

c. Result subtraction



b. Frame Current

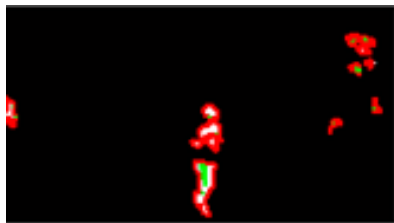

d. Blob detection

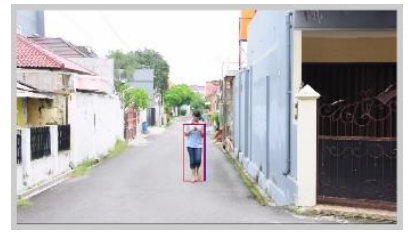

e. Objet Tracking using Kalman Filter

Fig. 3. Result Background-foreground process and tracking using kalman filter.

Mean square error [13] is applied to determine the accuracy of object tracking using Kalman Filter. MSE functions to calculate the different values from images which have experienced subtraction background with images that have been processed by Kalman Filter. The lower the value generated from MSE, the better the accuracy of object tracking. MSE can be measured by the following equation

$$
\operatorname{MSE}(G, F)=\frac{1}{Z W} \sum_{i=1}^{Z} \sum_{j=1}^{W}[G(i, j)-F(i, j)]
$$

The parameter $\mathrm{B}$ in the equation above shows the frame ground truth image, whereas $\mathrm{F}$ shows the experimental frame. The value of $\mathrm{Z}, \mathrm{W}$ is the size of image frame. In this experiment, two datasets were used as an evaluation of filter performance tested by the MSE equation. The results is shown in table 1 below:

Table 1. MSE result of dataset tracking with kalman filter.

\begin{tabular}{ll}
\hline Dataset & Average MSE Value \\
\hline Dataset1 & 34432.22 \\
Dataset2 & 342059.92 \\
\hline
\end{tabular}




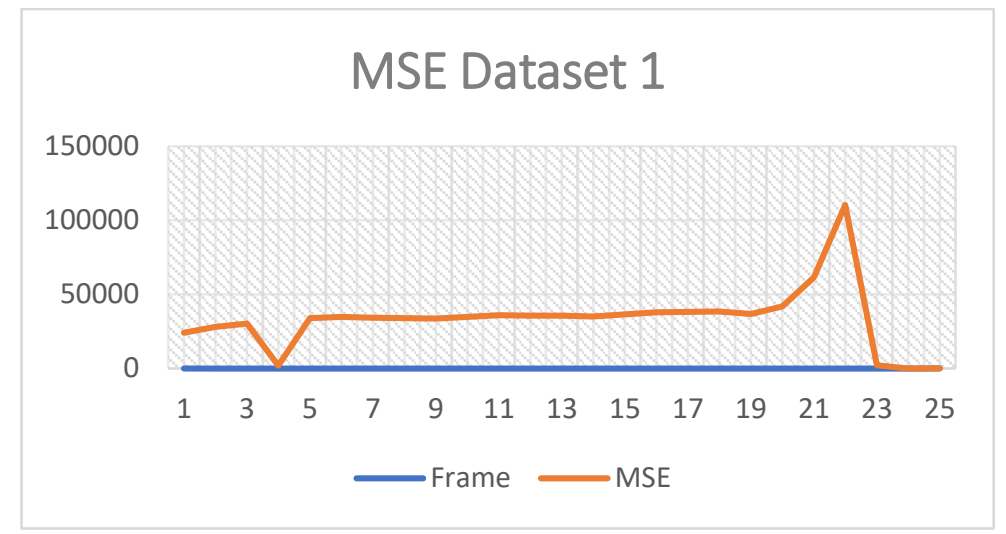

Fig. 4. MSE tracking object kalman filter.

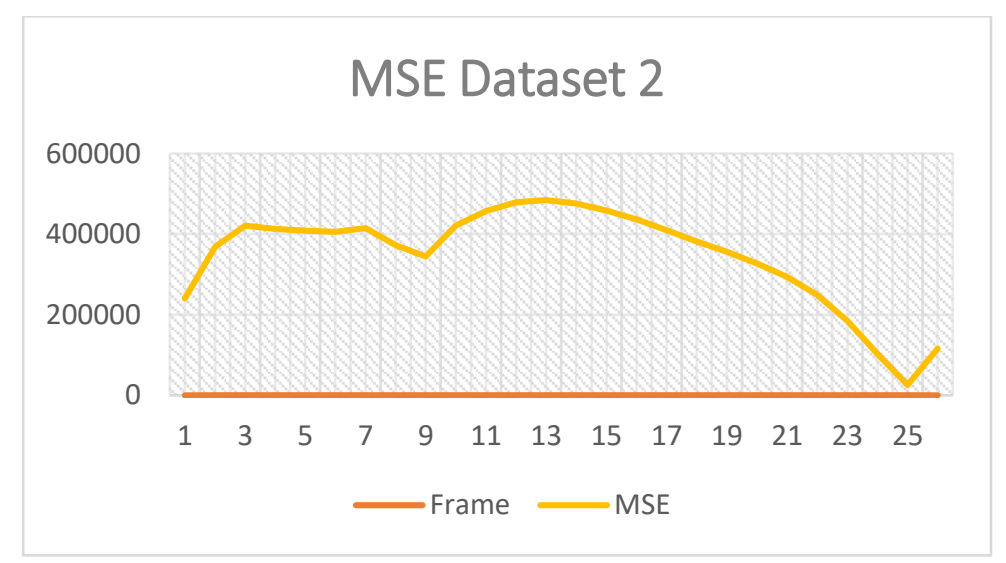

Fig. 5. MSE tracking object kalman filter.

\section{Conclusion}

In this paper, we proposed the Kalman filter method based on background subtraction to detect and track the movement of moving objects particularly to human objects. The experimental results showed that the detection process applying the background subtraction successfully detects objects and corrects detection results with median filter operations and morphology operations to obtain good results. Detected. When it is successfully detected, kalman filter could be applied to track the movement. Performance evaluation was performed by applying the mean square error. 


\section{Acknowledgments}

This research is supported by Ristekdikti Republik Indonesia.

\section{References}

[1] R. Aarthi, J. Amudha, K. Boomika a Anagha Varrier, „Detection of Moving Objects in Surveillance Video by Integrating Bottom-up approach with Knowledge Base,“ rev. International Conference on Information Security \& Privacy (ICISP2015), Nagpur, India, 2016.

[2] Y. Chaun, Z. Lihe, L. Huchuan, R. Xiang a Y. Ming-Hsuan, „Saliency Detection via Graph based Manifold Ranking," rev. Proceeding of IEEE Conference on Computer Vision and Pattern Recognition (CVPR 2013), Portland, 2013.

[3] S. Moch Arief, H. Mochamad a P. Mauridhi Hery, ,Adaptive threshold for background subtraction in moving object detection using Fuzzy c-Means clustering," rev. TENCON 2012, Cebu Philippines, 2012.

[4] B. Pouya , C. Sayed Ali a M. M. Musa bin, „Upper body tracking using KLT and Kalman filter,“ rev. Proceedings of the International Neural Network Society Winter Conference (INNS-WC2012), 2012.

[5] Z. Dali, Z. Bobai a W. Siye, „Mobile target indoor tracking based on Multi- D irection Weight Position Kalman Filter,“ Computer Networks, Elsevier, zv. 141, pp. 115-127, 2018.

[6] M. Azeem, „Modified background subtraction algorithm for motion detection in surveillance systems, “ Journal of American Arabic Academy for Science and Technology, zv. 1, \%1. vyd.2, pp. $112-123,2010$

[7] L. Alandkar a R. S, „Dealing Background Issues in Object Detection using GMM: A Survey,,“ Interntional Journal Comput. Appl., zv. 150, \%1. vyd.5, pp. 50-55, 2016.

[8] T. Aach, A. Kaup a R. Mester, „Change detection in image sequences using Gibbs random fields: a Bayesian approach,“ rev. the International Workshop on Intelligent Signal Processing and Communication Systems, Sendai, Japan, 1993.

[9] A. Irene a Bin Li, „Visual Object Tracking Based on Mean-shift and Particle-Kalman,“ rev. 2nd International Conference on Computer Science and Computational Intelligence 2017,, Bali Indonesia, 2017.

[10] S. Elhabian, E. Sayeed a S. Ahmed, „Moving object detection in spatial domain using background removal techniques-state-of-art.," Recent Patents on Computer Science, zv. 1, \%1. vyd.1, pp. 3854, 2008.

[11] M. K a K. K, „A New Hybrid Video Segmentation Algorithm using,“ International Journal of Computer Science, Zv. \%1 z \%2Vol. 9, Issue 2, No 1, \%1. vyd.Issue 2, No.1, pp. 229-237, 2012.

[12] K. Bhoyar a O. Kakde, „Color Image segmentation based on color histogram,“ International journal of Image Processing, zv. 6, \%1. vyd.3, pp. 283-293, 2010.

[13] B. Jose a M. G. Norberto , „Speed adaptation as Kalman filtering,“ Vision Reserach, Elsevier, zv. 48, p. 2485-2491, 2016.

[14] V. Kesari, S. Bikes Kumar a T. A S, „An Enhancement in Adaptive Median filter for Edge Preservation,“ rev. International Conference on Intelligent Computing, Communication \& Convergence, Bhubaneswar, Odisha, India, 2015. 